\title{
EchoGéo
}

$28 \mid 2014$

Police : les espaces de l'ordre, l'ordre en espace

\section{Polices et territoires}

Jean-Louis Chaléard

\section{OpenEdition}

Journals

Édition électronique

URL : https://journals.openedition.org/echogeo/13875

DOI : 10.4000/echogeo.13875

ISSN : 1963-1197

\section{Éditeur}

Pôle de recherche pour l'organisation et la diffusion de l'information géographique (CNRS UMR 8586)

Référence électronique

Jean-Louis Chaléard, «Polices et territoires », EchoGéo [En ligne], 28 | 2014, mis en ligne le 08 juillet 2014, consulté le 31 juillet 2021. URL : http://journals.openedition.org/echogeo/13875 ; DOI : https:// doi.org/10.4000/echogeo.13875

Ce document a été généré automatiquement le 31 juillet 2021.

EchoGéo est mis à disposition selon les termes de la licence Creative Commons Attribution - Pas d'Utilisation Commerciale - Pas de Modification 4.0 International (CC BY-NC-ND) 


\title{
Polices et territoires
}

\author{
Jean-Louis Chaléard
}

1 Ce numéro d'EchoGéo est intégralement consacré à la "police » et au "policier », à l'exception de la rubrique Sur le vif. Pendant longtemps, en dehors de quelques travaux pionniers, les géographes se sont peu intéressés à ce thème, alors que Michel Foucault a mis en évidence voilà plusieurs décennies le rôle des dispositifs de contrôle social et de surveillance dans nos sociétés et que les travaux sur la police et la gendarmerie se sont multipliés en histoire, en sociologie, en sciences politiques, etc. Les choses sont en train de changer, comme en témoigne, entre autres, le numéro récent (décembre 2013) du Bulletin de la Société Géographie de Liège sur le « crime mapping \& modelling ». Cette livraison d'Echogéo, coordonnée par Marie Redon et Armelle Choplin, s'inscrit dans ce mouvement.

2 Le thème est lourd, car chargé d'affectif, de visions diffusées par les médias par toujours fidèles à la réalité, et objet de controverses à propos de la sécurité quotidienne : séries télévisées policières en "prime time », longs développements sur les faits divers meurtriers dans les journaux télévisés, polémiques sur la mise en place de vidéo-surveillance dans les lieux publics, querelles entre majorité et opposition sur les chiffres de la délinquance, etc. Au-delà des présentations spectaculaires, des approches émotionnelles ou de l'instrumentalisation de peurs plus ou moins raisonnées, ce numéro essaie de prendre du recul et d'aborder le fait policier dans nos sociétés en privilégiant le rapport à l'espace, la construction réciproque du territoire et de la société derrière l'action de contrôle social et territorial.

Le dossier de la rubrique Sur le champ, «Espaces de l'ordre, l'ordre en place. Spatialités et pratiques policières » prend la question dans un sens très large. Il ne s'agit pas de s'intéresser seulement à la police, mais à l'ensemble des dispositifs de surveillance et de contrôle qui existent dans nos sociétés. Sans revenir sur la présentation de Marie Redon et Armelle Choplin en introduction du dossier, on peut ici insister sur quelques points. Tout d'abord les textes, par leurs entrées différentes, montrent que la sécurité et le maintien de l'ordre sont l'affaire d'acteurs multiples : policiers certes, mais aussi gendarmes, agents municipaux, parfois populations... Les espaces et territoires abordés couvrent un large éventail, permettant d'aller au-delà des cas particuliers. Les articles 
portent sur des exemples situés aussi bien en France que dans d'autres pays qu'ils soient au nord (Canada) ou au sud (Cambodge, Pérou...). Ils abordent tant les centres urbains que les périphéries ou les campagnes, les espaces «d'enfermement" (prisons...) comme les centres de formation ou d'entraînement des forces de police.

Ensuite, un volet important, abordé dans de nombreuses contributions, est celui des techniques ainsi que leur évolution (avec le recours croissant à la géolocalisation, aux SIG, etc.), qui rejaillissent sur les méthodes de contrôle du territoire. Quelques textes étudient plus spécifiquement ces questions, comme celui de Mélina Germes sur la cartographie policière ou celui de Anthony Goreau-Ponceaud et Émilie PonceaudGoreau sur le centre de formation de Saint Astier. Mais l'espace ne produit-il pas aussi des formes particulières de contrôle policier? L'interrogation est approfondie avec l'étude des espaces pénitentiaires en Guadeloupe par Marie Redon et Delphine Grancher, qui mettent en évidence les singularités insulaires et leurs limites.

Enfin, l'ensemble débouche sur une analyse de la complexité des relations territoirespratiques policières ou territoires-maintien de l'ordre à différentes échelles. Gabriel Fauveaud s'intéresse à la relation entre le fait policier et la production d'un territoire métropolitain, celui de Phnom Penh. Au-delà du rôle instrumental de la police dans le contrôle de la pauvreté ou le maintien d'un système politico-économique, l'auteur met en évidence la capacité des forces de l'ordre à produire du territoire, en jouant notamment avec le « désordre urbain ». À un niveau différent, Igor Lefevre s'interroge sur les ressorts spatiaux de l'insécurité en région Midi-Pyrénées, ainsi que sur les politiques publiques mises en œuvre face à des situations perçues par les acteurs locaux comme risquées ou dangereuses pour la sécurité des habitants. Le "marketing urbain », dans le cadre de la concurrence mondiale entre les grandes villes, affecte les politiques de contrôle de l'espace en ville. Les populations «marginales » sont l'objet d'un contrôle policier important, comme les SDF à Québec (Catherine Chesnay, Céline Bellot et Marie- Eve Sylvestre). Les quartiers populaires sont en première ligne : à Rio de Janeiro les autorités réaffirment leur pouvoir sur les favelas (Justine Ninin). Pour cela des nouvelles formes de contrôle peuvent être mises en place: à Lima des organisations populaires ont été créées pour seconder la police dans les quartiers défavorisés (Camille Boutron). Ainsi, l'analyse renvoie à des questions qui vont au-delà du fait policier sur la construction des territoires et la société en général.

6 En parallèle au dossier Sur le champ, la rubrique Sur le métier s'interroge sur les pratiques spatiales des gendarmes et des policiers à partir de l'interview de deux anciens gendarmes et d'un policier de la Préfecture de Police de Paris. La carte et le territoire sont au cœur des réflexions et des pratiques : cartes qui sont des outils de base pour se repérer, pour localiser les secteurs dangereux, pour planifier les actions à mener. Les témoignages révèlent une analyse fine du territoire de la part des services d'ordre, des activités qui varient selon les heures (Pigalle la nuit n'est pas Pigalle le jour...). Espace et territoire sont au cœur du métier mais aussi au cœur de la vie quotidienne des agents et de l'évolution des carrières : changements d'affectations qui retentissent sur la vie familiale et professionnelle, difficultés à vivre à Paris quand on y est en poste, mais nécessité de bien connaître le quartier où on travaille...

7 Dans la perspective de ce numéro, il était logique que la rubrique Sur l'écrit s'intéresse au roman policier. Martine Berger décrypte la société et la ville sud-africaine, ainsi que leur évolution à travers huit romans de Deon Meyer, grand auteur sud-africain. Le roman policier est-il une entrée privilégiée pour voir et analyser la réalité sociale et 
spatiale? L'interview de Michel Bussi, géographe et écrivain de romans policiers, montre que l'intérêt de la géographie est peut-être moins là où on pourrait l'attendre, dans la précision de la description des lieux, que dans leur transformation en les chargeant d'émotion. Réalité oui, mais transformée par la magie des auteurs.

8 L'article de de Jorick Perrin dans la rubrique Sur l'image complète les études de cette livraison d'ÉchoGéo sur le thème de la police et de ses pratiques spatiales en montrant comment l'analyse statistique et cartographique de la délinquance peut révéler des recompositions dans les relations villes-campagnes. Très présentes dans le discours médiatico-politique, les caractéristiques de la délinquance sont peu étudiées scientifiquement. Cet article essaie de palier ce déficit en présentant des pistes de lecture de l'influence de la ville sur les territoires périurbains et ruraux.

Seule la rubrique Sur le vif échappe à la thématique de la police et de la gendarmerie (quoi que...) et s'intéresse à deux cas latino-américains. Au moment de la Coupe du Monde de football, il était nécessaire de faire le point sur la nouvelle puissance brésilienne. Bernard Bret nous présente les ambitions du géant sud-américain, ses performances économiques et l'évolution de sa société. Les troubles de 2013 et ceux qui ont précédé le mondial de football montrent les faiblesses du pays, mais aussi son évolution qui se traduit dans l'origine des manifestants (plutôt petites classes moyennes), ou le droit effectif à manifester, ce qui tendrait à montrer que la démocratie est validée. On retrouve certains de ces thèmes dans l'article de Julien Robotier qui analyse des violences que connaît le Venezuela depuis février 2014. L'auteur explique comment ces manifestations sont à la croisée de deux tendances que l'on retrouve fréquemment en Amérique du Sud: la montée en puissance d'exigences sociales diverses et nouvelles, et des États encore fragiles. On pourra observer que les questions de police, centrales dans ce numéro, sont présentes également dans ces deux articles. C'est sans doute parce que les réflexions sur l'ordre public et son maintien touchent intimement à celles sur la démocratie. 